\title{
SUPPORTING INFORMATION Are ring currents still useful to rationalize the benzene proton magnetic shielding?
}

\author{
Rosario G. Viglione ${ }^{\dagger}$, Paolo Lazzeretti ${ }^{\ddagger}$ and Riccardo Zanasi ${ }^{\dagger}, *$ \\ ${ }^{\dagger}$ Dipartimento di Chimica, Università degli Studi di Salerno, via S. Allende, \\ 84081 Baronissi (SA), Italy \\ ${ }^{\ddagger}$ Dipartimento di Chimica, Università degli Studi di Modena, via G. Campi \\ 183, 41100 Modena, Italy
}

Molecular geometries. Experimental bond lengths and angles have been used in the calculations. Molecules have been oriented in such a way that: (i) the molecular plane of benzene and ethene concides with the $x y$ cartesian plane; (ii) the molecular axis of ethyne is parallel to the $z$ cartesian axis; (iii) in all cases the origin of the cartesian coordinate system is chosen to be in the center of mass (CM). This correspond to the following cartesian coordinates, which are reported in Bohr only for the symmetry unique atoms.

\begin{tabular}{cccccc}
\hline Molecule & Symmetry & Atom & $x$ & $y$ & $z$ \\
\hline Benzene & $D_{6 h}$ & $\mathrm{C}$ & 2.6362200 & 0.0000000 & 0.0000000 \\
& & $\mathrm{H}$ & 4.6866100 & 0.0000000 & 0.0000000 \\
Ethene & $D_{2 h}$ & $\mathrm{C}$ & 0.0000000 & 1.2651718 & 0.0000000 \\
& & $\mathrm{H}$ & 1.7556499 & 2.3242475 & 0.0000000 \\
Ethyne & $D_{\infty h}$ & $\mathrm{C}$ & 0.0000000 & 0.0000000 & 1.1366704 \\
& & $\mathrm{H}$ & 0.0000000 & 0.0000000 & 3.1416700 \\
\hline
\end{tabular}

Basis set. The basis set is the same for all molecules and it is a non contracted (13s10p5d2f/8s4p1d) set of Gaussian functions, whose exponents are reported in the following table. 


\begin{tabular}{crr}
\hline Type & \multicolumn{1}{c}{$\mathrm{C}$} & \multicolumn{1}{c}{$\mathrm{H}$} \\
\hline $\mathrm{s}$ & 50557.500670 & 402.009948 \\
& 7524.785601 & 60.241959 \\
& 1694.327599 & 13.732173 \\
& 472.822789 & 3.904505 \\
& 151.710752 & 1.282709 \\
& 53.918746 & 0.465544 \\
& 20.659311 & 0.181120 \\
& 8.383976 & 0.072791 \\
& 3.577015 & \\
& 1.547118 & \\
& 0.613013 & \\
& 0.246068 & \\
$\mathrm{p}$ & 0.099087 & \\
& 1512.900000 & 6.269000 \\
& 355.100000 & 2.292000 \\
& 83.333155 & 0.838000 \\
& 19.557611 & 0.292000 \\
& 6.080365 & \\
& 2.179317 & \\
& 0.865150 & \\
& 0.361944 & \\
& 0.154740 & \\
$\mathrm{~d}$ & 0.065429 & \\
& 5.262000 & 1.057000 \\
& 1.848000 & \\
& 0.649000 & \\
& 0.228000 & \\
& 0.080000 & \\
& 1.419000 & \\
& 0.485000 & \\
& & \\
& &
\end{tabular}

SCF Energies. The computed SCF energies in Hartree are:

\begin{tabular}{cc}
\hline Molecule & \multicolumn{1}{c}{ SCF Energy } \\
\hline Benzene & -230.7958572447 \\
Ethene & -78.0696422424 \\
Ethyne & -76.8553562477 \\
\hline
\end{tabular}


Magnetic properties. CO (common origin) and CTOCD (continuous transformation of the origin of the current density, in all its variants, i.e. diamagneticzero DZ1, paramagnetic-zero PZ1, dumped diamagnetic-zero DZ2 and dumped paramagnetic-zero PZ2) magnetizabilities and nuclear magnetic shieldings must be the same in the limit of a complete basis set calculation for any given model Hamiltonian. The following tables collect the isotropic magnetizabilities and absolute ${ }^{13} \mathrm{C}$ and ${ }^{1} \mathrm{H}$ magnetic shieldings computed at the CHF (coupled HartreeFock) level of theory.

Isotropic magnetizabilities in ppm (cgs) au.

\begin{tabular}{cccccc}
\hline Molecule & CO=CM & DZ1 & PZ1 & DZ2 & PZ2 \\
\hline Benzene & -678.549 & -675.628 & -676.291 & -675.577 & -676.144 \\
Ethene & -240.226 & -239.160 & -239.658 & -239.128 & -239.578 \\
Ethyne & -259.864 & -259.407 & -259.682 & -259.382 & -259.644 \\
\hline
\end{tabular}

Isotropic absolute ${ }^{13} \mathrm{C}$ magnetic shieldings in ppm.

\begin{tabular}{crrrrr}
\hline Molecule & $\mathrm{CO}=\mathrm{C} 1$ & \multicolumn{1}{c}{ DZ1 } & \multicolumn{1}{c}{ PZ1 } & \multicolumn{1}{c}{ DZ2 } & \multicolumn{1}{c}{ PZ2 } \\
\hline Benzene & 52.366 & 51.873 & 52.104 & 52.196 & 52.241 \\
Ethene & 56.128 & 55.693 & 55.977 & 56.010 & 56.080 \\
Ethyne & 114.703 & 114.258 & 114.620 & 114.585 & 114.695 \\
\hline
\end{tabular}

Isotropic absolute ${ }^{1} \mathrm{H}$ magnetic shieldings in ppm.

\begin{tabular}{crcccc}
\hline Molecule & $\mathrm{CO}=\mathrm{H} 1$ & DZ1 & PZ1 & DZ2 & PZ2 \\
\hline Benzene & 24.214 & 24.005 & 24.112 & 24.098 & 24.111 \\
Ethene & 26.063 & 25.864 & 25.990 & 25.986 & 26.006 \\
Ethyne & 30.378 & 30.185 & 30.317 & 30.298 & 30.332 \\
\hline
\end{tabular}


CHF/CTOCD-DZ out-of-plane $\pi$-electron shielding domain (in ppm) for benzene, see Fig. 1. Points (cartesian coordinates in Bohr relative to $\mathrm{CM}$ ) are on the $x z$ plane.

\begin{tabular}{|c|c|c|c|c|c|c|c|c|c|c|c|}
\hline$x$ & $z$ & $\sigma_{\|}$ & $x$ & $z$ & $\sigma_{\|}$ & $x$ & $z$ & $\sigma_{\|}$ & $x$ & $z$ & $\sigma_{\|}$ \\
\hline 0.0 & 0.0 & 37.260 & 0.0 & 0.5 & 37.194 & 0.0 & 1.0 & 36.327 & 0.0 & 1.5 & 33.761 \\
\hline 0.0 & 2.0 & 29.580 & 0.0 & 2.5 & 24.644 & 0.0 & 3.0 & 19.861 & 0.0 & 3.5 & 15.701 \\
\hline 0.0 & 4.0 & 12.314 & 0.0 & 4.5 & 9.660 & 0.0 & 5.0 & 7.618 & 0.0 & 5.5 & 6.057 \\
\hline 0.5 & 0.0 & 37.234 & 0.5 & 0.5 & 37.333 & 0.5 & 1.0 & 36.656 & 0.5 & 1.5 & 34.030 \\
\hline 0.5 & 2.0 & 29.621 & 0.5 & 2.5 & 24.519 & 0.5 & 3.0 & 19.641 & 0.5 & 3.5 & 15.474 \\
\hline 0.5 & 4.0 & 12.117 & 0.5 & 4.5 & 8.537 & 0.5 & 5.0 & 7.496 & 0.5 & 5.5 & 5.965 \\
\hline 1.0 & 0.0 & 36.876 & 1.0 & 0.5 & 37.610 & 1.0 & 1.0 & 37.430 & 1.0 & 1.5 & 34.464 \\
\hline 1.0 & 2.0 & 29.440 & 1.0 & 2.5 & 23.883 & 1.0 & 3.0 & 18.869 & 1.0 & 3.5 & 14.748 \\
\hline 1.0 & 4.0 & 11.511 & 1.0 & 4.5 & 9.038 & 1.0 & 5.0 & 7.136 & 1.0 & 5.5 & 5.696 \\
\hline 1.5 & 0.0 & 35.409 & 1.5 & 0.5 & 37.174 & 1.5 & 1.0 & 37.644 & 1.5 & 1.5 & 33.864 \\
\hline 1.5 & 2.0 & 27.981 & 1.5 & 2.5 & 22.167 & 1.5 & 3.0 & 17.268 & 1.5 & 3.5 & 13.414 \\
\hline 1.5 & 4.0 & 10.475 & 1.5 & 4.5 & 8.242 & 1.5 & 5.0 & 6.552 & 1.5 & 5.5 & 5.263 \\
\hline 2.0 & 0.0 & 31.494 & 2.0 & 0.5 & 34.814 & 2.0 & 1.0 & 34.858 & 2.0 & 1.5 & 30.018 \\
\hline 2.0 & 2.0 & 24.080 & 2.0 & 2.5 & 18.827 & 2.0 & 3.0 & 14.655 & 2.0 & 3.5 & 11.451 \\
\hline 2.0 & 4.0 & 9.025 & 2.0 & 4.5 & 7.185 & 2.0 & 5.0 & 5.779 & 2.0 & 5.5 & 4.697 \\
\hline 2.5 & 0.0 & 22.475 & 2.5 & 0.5 & 25.565 & 2.5 & 1.0 & 24.761 & 2.5 & 1.5 & 21.217 \\
\hline 2.5 & 2.0 & 17.336 & 2.5 & 2.5 & 13.945 & 2.5 & 3.0 & 11.188 & 2.5 & 3.5 & 9.003 \\
\hline 2.5 & 4.0 & 7.286 & 2.5 & 4.5 & 5.939 & 2.5 & 5.0 & 4.878 & 2.5 & 5.5 & 4.037 \\
\hline 3.0 & 0.0 & 9.028 & 3.0 & 0.5 & 8.573 & 3.0 & 1.0 & 9.635 & 3.0 & 1.5 & 10.003 \\
\hline 3.0 & 2.0 & 9.498 & 3.0 & 2.5 & 8.550 & 3.0 & 3.0 & 7.465 & 3.0 & 3.5 & 6.410 \\
\hline 3.0 & 4.0 & 5.457 & 3.0 & 4.5 & 4.631 & 3.0 & 5.0 & 3.927 & 3.0 & 5.5 & 3.339 \\
\hline 3.5 & 0.0 & 1.287 & 3.5 & 0.5 & 0.101 & 3.5 & 1.0 & 0.270 & 3.5 & 1.5 & 1.885 \\
\hline 3.5 & 2.0 & 3.278 & 3.5 & 2.5 & 4.009 & 3.5 & 3.0 & 4.200 & 3.5 & 3.5 & 4.064 \\
\hline 3.5 & 4.0 & 3.761 & 3.5 & 4.5 & 3.392 & 3.5 & 5.0 & 3.014 & 3.5 & 5.5 & 2.656 \\
\hline 4.0 & 0.0 & -1.790 & 4.0 & 0.5 & -2.459 & 4.0 & 1.0 & -2.769 & 4.0 & 1.5 & -1.735 \\
\hline 4.0 & 2.0 & -0.222 & 4.0 & 2.5 & 1.029 & 4.0 & 3.0 & 1.821 & 4.0 & 3.5 & 2.228 \\
\hline 4.0 & 4.0 & 2.360 & 4.0 & 4.5 & 2.326 & 4.0 & 5.0 & 2.200 & 4.0 & 5.5 & 2.031 \\
\hline 4.5 & 0.0 & -2.760 & 4.5 & 0.5 & -3.071 & 4.5 & 1.0 & -3.303 & 4.5 & 1.5 & -2.754 \\
\hline 4.5 & 2.0 & -1.665 & 4.5 & 2.5 & -0.535 & 4.5 & 3.0 & 0.362 & 4.5 & 3.5 & 0.966 \\
\hline 4.5 & 4.0 & 1.316 & 4.5 & 4.5 & 1.482 & 4.5 & 5.0 & 1.525 & 4.5 & 5.5 & 1.494 \\
\hline 5.0 & 0.0 & -2.856 & 5.0 & 0.5 & -2.995 & 5.0 & 1.0 & -3.081 & 5.0 & 1.5 & -2.746 \\
\hline 5.0 & 2.0 & -2.029 & 5.0 & 2.5 & -1.181 & 5.0 & 3.0 & -0.409 & 5.0 & 3.5 & 0.190 \\
\hline 5.0 & 4.0 & 0.604 & 5.0 & 4.5 & 0.860 & 5.0 & 5.0 & 0.999 & 5.0 & 5.5 & 1.055 \\
\hline 5.5 & 0.0 & -2.599 & 5.5 & 0.5 & -2.646 & 5.5 & 1.0 & -2.644 & 5.5 & 1.5 & -2.413 \\
\hline 5.5 & 2.0 & -1.941 & 5.5 & 2.5 & -1.343 & 5.5 & 3.0 & -0.747 & 5.5 & 3.5 & -0.238 \\
\hline 5.5 & 4.0 & 0.153 & 5.5 & 4.5 & 0.429 & 5.5 & 5.0 & 0.608 & 5.5 & 5.5 & 0.713 \\
\hline 6.0 & 0.0 & -2.241 & 6.0 & 0.5 & -2.245 & 6.0 & 1.0 & -2.201 & 6.0 & 1.5 & -2.028 \\
\hline 6.0 & 2.0 & -1.708 & 6.0 & 2.5 & -1.317 & 6.0 & 3.0 & -0.851 & 6.0 & 3.5 & -0.447 \\
\hline 6.0 & 4.0 & -0.112 & 6.0 & 4.5 & 0.146 & 6.0 & 5.0 & 0.331 & 6.0 & 5.5 & 0.455 \\
\hline 6.5 & 0.0 & -1.892 & 6.5 & 0.5 & -1.880 & 6.5 & 1.0 & -1.822 & 6.5 & 1.5 & -1.686 \\
\hline 6.5 & 2.0 & -1.458 & 6.5 & 2.5 & -1.163 & 6.5 & 3.0 & -0.841 & 6.5 & 3.5 & -0.530 \\
\hline 6.5 & 4.0 & -0.257 & 6.5 & 4.5 & -0.033 & 6.5 & 5.0 & 0.140 & 6.5 & 5.5 & 0.266 \\
\hline 7.0 & 0.0 & -1.586 & 7.0 & 0.5 & -1.570 & 7.0 & 1.0 & -1.513 & 7.0 & 1.5 & -1.404 \\
\hline 7.0 & 2.0 & -1.236 & 7.0 & 2.5 & -1.021 & 7.0 & 3.0 & -0.784 & 7.0 & 3.5 & -0.546 \\
\hline 7.0 & 4.0 & -0.328 & 7.0 & 4.5 & -0.140 & 7.0 & 5.0 & 0.013 & 7.0 & 5.5 & 0.132 \\
\hline
\end{tabular}


CHF/CTOCD-DZ out-of-plane $\pi$-electron shielding domain (in ppm) for ethene, see Fig. 2. Points (cartesian coordinates in Bohr relative to $\mathrm{CM}$ ) are on the $y z$ plane.

\begin{tabular}{|c|c|c|c|c|c|c|c|c|c|c|c|}
\hline$y$ & $z$ & $\sigma_{\|}$ & $y$ & $z$ & $\sigma_{\|}$ & $y$ & $z$ & $\sigma_{\|}$ & $y$ & $z$ & $\sigma_{\|}$ \\
\hline 0.0 & 0.0 & 11.430 & 0.0 & 0.5 & 13.065 & 0.0 & 1.0 & 14.601 & 0.0 & 1.5 & 13.351 \\
\hline 0.0 & 2.0 & 10.540 & 0.0 & 2.5 & 7.691 & 0.0 & 3.0 & 5.421 & 0.0 & 3.5 & 3.782 \\
\hline 0.0 & 4.0 & 2.655 & 0.0 & 4.5 & 1.890 & 0.0 & 5.0 & 1.371 & & & \\
\hline 0.5 & 0.0 & 11.471 & 0.5 & 0.5 & 13.416 & 0.5 & 1.0 & 14.762 & 0.5 & 1.5 & 13.082 \\
\hline 0.5 & 2.0 & 10.130 & 0.5 & 2.5 & 7.338 & 0.5 & 3.0 & 5.169 & 0.5 & 3.5 & 3.619 \\
\hline 0.5 & 4.0 & 2.552 & 0.5 & 4.5 & 1.825 & 0.5 & 5.0 & 1.330 & & & \\
\hline 1.0 & 0.0 & 10.883 & 1.0 & 0.5 & 13.570 & 1.0 & 1.0 & 13.881 & 1.0 & 1.5 & 11.542 \\
\hline 1.0 & 2.0 & 8.704 & 1.0 & 2.5 & 6.283 & 1.0 & 3.0 & 4.464 & 1.0 & 3.5 & 3.168 \\
\hline 1.0 & 4.0 & 2.267 & 1.0 & 4.5 & 1.646 & 1.0 & 5.0 & 1.215 & & & \\
\hline 1.5 & 0.0 & 7.246 & 1.5 & 0.5 & 8.653 & 1.5 & 1.0 & 9.145 & 1.5 & 1.5 & 7.927 \\
\hline 1.5 & 2.0 & 6.197 & 1.5 & 2.5 & 4.646 & 1.5 & 3.0 & 3.424 & 1.5 & 3.5 & 2.513 \\
\hline 1.5 & 4.0 & 1.855 & 1.5 & 4.5 & 1.384 & 1.5 & 5.0 & 1.046 & & & \\
\hline 2.0 & 0.0 & 3.274 & 2.0 & 0.5 & 3.207 & 2.0 & 1.0 & 3.640 & 2.0 & 1.5 & 3.783 \\
\hline 2.0 & 2.0 & 3.428 & 2.0 & 2.5 & 2.860 & 2.0 & 3.0 & 2.285 & 2.0 & 3.5 & 1.788 \\
\hline 2.0 & 4.0 & 1.391 & 2.0 & 4.5 & 1.084 & 2.0 & 5.0 & 0.849 & & & \\
\hline 2.5 & 0.0 & 1.140 & 2.5 & 0.5 & 0.876 & 2.5 & 1.0 & 0.832 & 2.5 & 1.5 & 1.148 \\
\hline 2.5 & 2.0 & 1.384 & 2.5 & 2.5 & 1.414 & 2.5 & 3.0 & 1.302 & 2.5 & 3.5 & 1.131 \\
\hline 2.5 & 4.0 & 0.953 & 2.5 & 4.5 & 0.790 & 2.5 & 5.0 & 0.651 & & & \\
\hline 3.0 & 0.0 & 0.180 & 3.0 & 0.5 & 0.004 & 3.0 & 1.0 & -0.148 & 3.0 & 1.5 & 0.005 \\
\hline 3.0 & 2.0 & 0.284 & 3.0 & 2.5 & 0.501 & 3.0 & 3.0 & 0.608 & 3.0 & 3.5 & 0.627 \\
\hline 3.0 & 4.0 & 0.595 & 3.0 & 4.5 & 0.537 & 3.0 & 5.0 & 0.472 & & & \\
\hline 3.5 & 0.0 & -0.201 & 3.5 & 0.5 & -0.294 & 3.5 & 1.0 & -0.407 & 3.5 & 1.5 & -0.358 \\
\hline 3.5 & 2.0 & -0.173 & 3.5 & 2.5 & 0.035 & 3.5 & 3.0 & 0.196 & 3.5 & 3.5 & 0.293 \\
\hline 3.5 & 4.0 & 0.335 & 3.5 & 4.5 & 0.340 & 3.5 & 5.0 & 0.325 & & & \\
\hline 4.0 & 0.0 & -0.322 & 4.0 & 0.5 & -0.366 & 4.0 & 1.0 & -0.429 & 4.0 & 1.5 & -0.413 \\
\hline 4.0 & 2.0 & -0.305 & 4.0 & 2.5 & -0.154 & 4.0 & 3.0 & -0.011 & 4.0 & 3.5 & 0.096 \\
\hline 4.0 & 4.0 & 0.164 & 4.0 & 4.5 & 0.199 & 4.0 & 5.0 & 0.212 & & & \\
\hline 4.5 & 0.0 & -0.334 & 4.5 & 0.5 & -0.353 & 4.5 & 1.0 & -0.381 & 4.5 & 1.5 & -0.371 \\
\hline 4.5 & 2.0 & -0.306 & 4.5 & 2.5 & -0.205 & 4.5 & 3.0 & -0.099 & 4.5 & 3.5 & -0.007 \\
\hline 4.5 & 4.0 & 0.061 & 4.5 & 4.5 & 0.106 & 4.5 & 5.0 & 0.131 & & & \\
\hline 5.0 & 0.0 & -0.305 & 5.0 & 0.5 & -0.312 & 5.0 & 1.0 & -0.320 & 5.0 & 1.5 & -0.309 \\
\hline 5.0 & 2.0 & -0.267 & 5.0 & 2.5 & -0.201 & 5.0 & 3.0 & -0.125 & 5.0 & 3.5 & -0.055 \\
\hline 5.0 & 4.0 & 0.003 & 5.0 & 4.5 & 0.046 & 5.0 & 5.0 & 0.075 & & & \\
\hline
\end{tabular}


CHF/CTOCD-DZ out-of-plane $\pi$-electron shielding domain (in ppm) for ethyne, see Fig. 3. Points (cartesian coordinates in Bohr relative to $\mathrm{CM}$ ) are on the $x z$ plane.

\begin{tabular}{|c|c|c|c|c|c|c|c|c|c|c|c|}
\hline$x$ & $z$ & $\sigma_{\|}$ & $x$ & $z$ & $\sigma_{\|}$ & $x$ & $z$ & $\sigma_{\|}$ & $x$ & $z$ & $\sigma_{\|}$ \\
\hline 0.0 & 0.0 & 32.181 & 0.0 & 0.5 & 61.434 & 0.0 & 1.0 & 55.563 & 0.0 & 1.5 & 44.653 \\
\hline 0.0 & 2.0 & 32.385 & 0.0 & 2.5 & 21.556 & 0.0 & 3.0 & 14.132 & 0.0 & 3.5 & 9.547 \\
\hline 0.0 & 4.0 & 6.630 & 0.0 & 4.5 & 4.703 & 0.0 & 5.0 & 3.426 & & & \\
\hline 0.5 & 0.0 & 57.039 & 0.5 & 0.5 & 56.430 & 0.5 & 1.0 & 51.774 & 0.5 & 1.5 & 41.733 \\
\hline 0.5 & 2.0 & 29.779 & 0.5 & 2.5 & 20.020 & 0.5 & 3.0 & 13.352 & 0.5 & 3.5 & 9.094 \\
\hline 0.5 & 4.0 & 6.336 & 0.5 & 4.5 & 4.527 & 0.5 & 5.0 & 3.317 & & & \\
\hline 1.0 & 0.0 & 37.122 & 1.0 & 0.5 & 36.246 & 1.0 & 1.0 & 33.147 & 1.0 & 1.5 & 27.579 \\
\hline 1.0 & 2.0 & 21.008 & 1.0 & 2.5 & 15.225 & 1.0 & 3.0 & 10.831 & 1.0 & 3.5 & 7.677 \\
\hline 1.0 & 4.0 & 5.509 & 1.0 & 4.5 & 4.038 & 1.0 & 5.0 & 3.023 & & & \\
\hline 1.5 & 0.0 & 17.630 & 1.5 & 0.5 & 16.845 & 1.5 & 1.0 & 15.201 & 1.5 & 1.5 & 13.580 \\
\hline 1.5 & 2.0 & 11.784 & 1.5 & 2.5 & 9.641 & 1.5 & 3.0 & 7.538 & 1.5 & 3.5 & 5.768 \\
\hline 1.5 & 4.0 & 4.390 & 1.5 & 4.5 & 3.362 & 1.5 & 5.0 & 2.601 & & & \\
\hline 2.0 & 0.0 & 5.875 & 2.0 & 0.5 & 5.657 & 2.0 & 1.0 & 5.389 & 2.0 & 1.5 & 5.470 \\
\hline 2.0 & 2.0 & 5.593 & 2.0 & 2.5 & 5.338 & 2.0 & 3.0 & 4.722 & 2.0 & 3.5 & 3.972 \\
\hline 2.0 & 4.0 & 3.253 & 2.0 & 4.5 & 2.633 & 2.0 & 5.0 & 2.127 & & & \\
\hline 2.5 & 0.0 & 0.453 & 2.5 & 0.5 & 0.524 & 2.5 & 1.0 & 0.838 & 2.5 & 1.5 & 1.457 \\
\hline 2.5 & 2.0 & 2.142 & 2.5 & 2.5 & 2.580 & 2.5 & 3.0 & 2.681 & 2.5 & 3.5 & 2.533 \\
\hline 2.5 & 4.0 & 2.261 & 2.5 & 4.5 & 1.955 & 2.5 & 5.0 & 1.661 & & & \\
\hline 3.0 & 0.0 & -1.569 & 3.0 & 0.5 & -1.423 & 3.0 & 1.0 & -0.991 & 3.0 & 1.5 & -0.326 \\
\hline 3.0 & 2.0 & 0.402 & 3.0 & 2.5 & 0.994 & 3.0 & 3.0 & 1.355 & 3.0 & 3.5 & 1.496 \\
\hline 3.0 & 4.0 & 1.484 & 3.0 & 4.5 & 1.384 & 3.0 & 5.0 & 1.245 & & & \\
\hline 3.5 & 0.0 & -2.055 & 3.5 & 0.5 & -1.921 & 3.5 & 1.0 & -1.545 & 3.5 & 1.5 & -0.994 \\
\hline 3.5 & 2.0 & -0.384 & 3.5 & 2.5 & 0.161 & 3.5 & 3.0 & 0.563 & 3.5 & 3.5 & 0.807 \\
\hline 3.5 & 4.0 & 0.919 & 3.5 & 4.5 & 0.937 & 3.5 & 5.0 & 0.900 & & & \\
\hline 4.0 & 0.0 & -1.938 & 4.0 & 0.5 & -1.836 & 4.0 & 1.0 & -1.553 & 4.0 & 1.5 & -1.141 \\
\hline 4.0 & 2.0 & -0.676 & 4.0 & 2.5 & -0.236 & 4.0 & 3.0 & 0.121 & 4.0 & 3.5 & 0.375 \\
\hline 4.0 & 4.0 & 0.530 & 4.0 & 4.5 & 0.607 & 4.0 & 5.0 & 0.628 & & & \\
\hline 4.5 & 0.0 & -1.642 & 4.5 & 0.5 & -1.570 & 4.5 & 1.0 & -1.369 & 4.5 & 1.5 & -1.074 \\
\hline 4.5 & 2.0 & -0.734 & 4.5 & 2.5 & -0.400 & 4.5 & 3.0 & -0.110 & 4.5 & 3.5 & 0.115 \\
\hline 4.5 & 4.0 & 0.273 & 4.5 & 4.5 & 0.371 & 4.5 & 5.0 & 0.423 & & & \\
\hline 5.0 & 0.0 & -1.336 & 5.0 & 0.5 & -1.286 & 5.0 & 1.0 & -1.146 & 5.0 & 1.5 & -0.939 \\
\hline 5.0 & 2.0 & -0.664 & 5.0 & 2.5 & -0.447 & 5.0 & 3.0 & -0.222 & 5.0 & 3.5 & -0.034 \\
\hline 5.0 & 4.0 & 0.108 & 5.0 & 4.5 & 0.208 & 5.0 & 5.0 & 0.271 & & & \\
\hline
\end{tabular}

